\title{
Der Nerv der Dinge
}

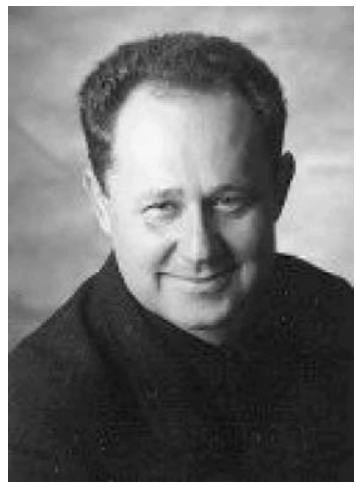

Prof. Dr. med. Manfred Wildner
Der „Nerv der Dinge“ - was das sein mag? Die Antwort auf diese Frage lautet von alters her eintönig gleich: das Geld. Der Aphorismus wird Bion von Borysthenes (335-225 v. Chr.) zugeschrieben, welcher zur altgriechischen Philosophenschule der Zyniker gehörte. Gemeint ist in dieser aus der Antike überkommenen Redewendung in einem modernen anatomischen Verständnis mit „Nerv“ allerdings mehr die Sehne als das Neuron: Also das, was der Sache Schwung gibt. Noch ein weiterer Aphorismus ist von ihm erhalten, diesmal über die Auswirkungen des Geldes auf einen Mann: „Er hat kein Vermögen an sich gebracht, das Vermögen hat ihn zu sich genommen!“ In unseren modernen Zeiten singt Liza Minelli in dem Film „Cabaret“ im gleichen Sinn: Money makes the world go round - Geld bewegt die Welt.

Da das Gesundheitswesen durchaus Teil dieser Welt ist, könnte man die Frage stellen, was denn eigentlich diesem Zauberstoff „Geld“ diese besonderen Kräfte verleiht. Etymologisch mit dem indogermanischen Gold verwandt, bedeutete Geld im Althochdeutschen zunächst so viel wie Vergeltung oder Vergütung und meinte in einem kultischen Gebrauch das, womit Buße und Opfer entrichtet werden konnte. Hier kann ein Bogen geschlagen werden zu den heute üblichen „Geldbußen“ bei Ordnungswidrigkeiten. Seine allgemeine Funktion im Wirtschaftsleben ist inzwischen sehr viel pragmatischer: Es ist hauptsächlich ein Zwischentauschmittel, hat also die Rolle, in einem Tauschgeschäft als Wert erhaltendes Zahlungsmittel mit weit verbreiteter Akzeptanz zu fungieren. Es ist seinen Vorläufern wie Getreide, Muscheln oder Kauri-Schnecken vor allem durch seinen relativ geringen Platzbedarf und seine doch recht große Haltbarkeit, zumindest in Form von Gold- oder Silbermünzen, überlegen.

A propos Kauri-Schnecken: Einige Arten dieser Meeresschnecke, wie die Cypraea moneta, galten lange Zeit in Afrika, Ost- und Südasien sowie der Südsee als allgemein anerkanntes Zahlungsmittel. Der Weltumsegler Bobby Schenk erzählt in einem seiner Bücher die Geschichte eines Bündels dieser wertvollen Meeresmuscheln, welche als Zahlungsmittel im Warenaustausch zwischen Südsee-Stämmen verwendet wurden [1]. Nachdem der eine Stamm die begehrten Waren geliefert hatte, wurden die wertvollen Muscheln in einer feierlichen Prozession durch einen Bach getragen - und gingen dort prompt verloren. Was tun? Die ortsansässigen Ökonomen hatten offenbar rasch ein Lösungskonzept zur Hand: Das verloren gegangene Zahlungsmittel wurde fortan als „verlorene Muscheln“ im allgemeinen Gedächtnis erhalten und auch als solche im Warenver- kehr weiter benutzt. Findige Burschen, diese Ökonomen. Unverzichtbare Voraussetzung ist allerdings ein hohes Maß an gesellschaftlicher Stabilität und gegenseitigem Vertrauen („Kredit“). Findet sich hier nicht eine bedenkenswerte Analogie zur heutigen Finanzwirtschaft? Ist es nicht vor allem das Vertrauen, was dem Zwischentauschmittel Geld seinen Wert gibt? Und damit den wirtschaftlichen Austausch, gerade auch im Gesundheitswesen mit seinen vielfältigen, komplizierten Dienstleistungen und Investitionsgütern, so reibungslos und elegant verlaufen lässt? Letztlich „arbeitet" Geld in seinen modernen Formen eben nicht bzw. nur bedingt. Es liegt entweder als farbig bedrucktes Papier in unseren Geldbörsen oder Bankschließfächern oder beult als Kuper- oder sonstige Metalllegierung die Hosentaschen und Handtaschen mehr oder weniger stark aus. Mit Ausnahme von Gold- und Silbermünzen ist ihr potentieller Gebrauchswert eher gering. Einen Kranken pflegen kann weder ein Einhundert- noch ein EintausendEuro-Schein, auch ein fälschungssicherer 5-EuroSchein vermag nicht eine Masernimpfung zu ersetzen und letztlich lässt sich der Anspruch auf Gegenleistungen in dem Zwischentauschmittel Geld nur solange speichern, wie andere Menschen sich darauf einlassen. Geld ist immer Kredit, also Vertrauen darauf, dass man etwas dafür bekommt.

Dieser zuletzt dargelegte Zusammenhang hat durchaus Relevanz für Überlegungen zur nachhaltigen Sicherung unserer Gesundheits- und Sozialsysteme. Kranken- und Rentenversicherungen sind in Versicherungssystemen wie dem deutschen Bismarck-System überwiegend als Umlage-Systeme konstruiert: Hier funktioniert das Geld vor allem als Verteilungsinstrument der verfügbaren Arbeitskraft und Produktivmittel im Gesundheitswesen an die bedürftigen Mitglieder einer versicherten Gemeinschaft. Das System funktioniert also eben nicht wie ein Sparkonto im herkömmlichen Sinne, auf welches im Lauf des Lebens eingezahlt wird und aus welchem aufgrund des so angesparten Kapitalstockes dann im weiteren Lebensverlauf Auszahlungen vorgenommen werden können. Die Altersrückstellungen in der PKV und die kapitalgedeckten Altersvorsorgemodelle sind eher Ausnahmen als die Regel. Versuche in diese Richtung sind in anderen Ländern durchaus im größeren Stil gemacht worden, z.B. mit dem 1984 im Gesundheitswesen von Singapur verpflichtend eingeführten Health Savings Account („Medisave“). Beachtlich ist die Auswirkung dieses Systems, bei welchem die Teilnehmer steuerfreie Einzahlungen auf ein persönliches Konto zur Begleichung von Gesundheitsausgaben leisten können, auf die aktuellen Gesamtausgaben für medizinische Leistun- 
gen [2]. Diese liegen als Anteil des Bruttosozialprodukts deutlich niedriger als bei anderen ökonomisch entwickelten Gesellschaften. Allerdings ist die Bevölkerung in Singapur demografisch jung.

In einer alternden Gesellschaft hat der Gedanke eines Kapitalstockes, also eines Zwischenspeichers, als vordergründig greifbare Sicherheit für Leistungen im späteren Lebensalter genauso Anfälligkeiten wie ein Umlagesystem. Wenn nur noch vergleichsweise wenig Leistungserbringer bereit sind, Dienstleistungen wie die Altenpflege zu übernehmen, wird auch viel Geld nicht ohne weiteres zusätzliche Hände schaffen können oder eben nur um einen hohen Preis. Solche bei einem ungünstigen Verhältnis von Leistungserbringern und bedürftigen Mitgliedern einer Gemeinschaft im zeitlichen Verlauf erhöhten Preise bringen den eigentlichen Vorsorgegedanken dann genauso in Schwierigkeiten wie ein Umlageverfahren. Schon 1952 formulierte der Sozialwissenschaftler Gerhard Mackenroth diesen Zusammenhang als These - dass nämlich die Sozialausgaben einer Volkswirtschaft aus dem Volkseinkommen der laufenden Periode zu erbringen sind [3].

Doch gehen wir einen Schritt zurück und betrachten Geld in seiner hauptsächlichen Funktion als Zwischentauschmittel oder Zwischenspeicher für eine ökonomische Leistungsbereitschaft bzw. -fähigkeit weiter. Diese kann entweder in Form von vorhandenem Vermögen (assets) vorliegen oder aber in Form von derzeitiger und zukünftiger Produktivität und Wertschöpfung. Welcher Zusammenhang besteht zwischen solchen ökonomischen Größen und Gesundheit? Ein Bericht der Weltgesundheitsorganisation ist diesem Zusammenhang nachgegangen [4]. Darin wurde der Einfluss der Gesundheit auf die ökonomische Entwicklung untersucht wohl gemerkt in dieser Richtung - und es wurden Auswirkungen in 3 Zusammenhängen gesehen. Zum ersten erhöht die Reduktion von vermeidbaren Erkrankungen die gesunde Lebenserwartung, was erhebliche Auswirkungen auf die gesellschaftliche Wohlfahrt hat. Die Produktivität der berufstätigen Bevölkerungsanteile bleibt so erhalten bzw. wird gesteigert und gerade bei ausbildungsintensiven Berufen ist der „return on investment“, also die Lebensarbeitszeit im Anschluss an die Ausbildung, länger gegeben. Gleichzeitig ist die Belastung durch wirtschaftlich unproduktive Arbeitsausfälle, Krankheitslasten und Todesfälle reduziert. Ökonomische Wachstumsschübe waren daher in einer historischen Betrachtung häufig mit positiven Entwicklungen im Bereich der Versorgung mit Nahrungsmitteln, der öffentlichen Gesundheit und der Bekämpfung von Krankheiten verknüpft. Darüber hinaus kann der persönliche Nutzwert eines gesunden Lebens bei den Menschen, die davon profitieren, den reinen Geldwert einer ökonomischen Betrachtung noch einmal um ein Vielfaches übersteigen.

Ein zweiter Vermittlungspfad ist der Einfluss von Gesundheit auf das elterliche Erziehungsverhalten: Bei niedriger Kindersterblichkeit werden in der Regel weniger Kinder geboren, in welche dann gleichzeitig auch in höherem Maße investiert wird, z. B. in Form von Bildungschancen. Eine niedrige Abhängigkeitsquote Minderjähriger stimuliert gleichzeitig den Verbrauch einer Gesellschaft in vielen anderen Bereichen, was zu einem im Allgemeinen erwünschten Wirtschaftswachstum beiträgt. Zum Dritten trägt ein guter Gesundheitszustand zu einer größeren wirtschaftlichen Attraktivität einer Gemeinschaften bzw. einer Region bei, was wiederum die wirtschaftliche Prosperität fördert. „Wer hat, dem wird gegeben“, lautet ja das Matthäus-Prinzip. Die korrespondierende Frage, nämlich welchen Einfluss wirtschaftlicher Reichtum auf die Gesundheit hat, ist ebenfalls differenziert zu betrachten. Zweifelsohne stellen materielle und personelle Gesundheitsressourcen, wie die Verfügbarkeit und der Zugang zu Krankenhäusern, spezialisierten Behandlungs- und
Diagnosezentren, Rehabilitationsmaßnahmen und auch der ambulanten ärztlichen und auch pflegerischen Versorgung eine wichtige gesundheitliche Ressource dar. Gleichzeitig zeigt sich auch, dass ein „Reichtum“ an Wissen und Bildung größere gesundheitliche Effekte hat als der rein materielle Reichtum. Aus internationalen Studien sind Beispiele bekannt, bei denen Länder bzw. regionale Gemeinschaften, welche über relativ geringe materielle Ressourcen, jedoch über hervorragende Bildungssysteme verfügten, einen unerwarteten guten gesundheitlichen Gesamtzustand ihrer Bevölkerungen ausweisen: Costa Rica in Mittelamerika, Kerala in Indien und andere mehr.

Und auch noch ein Drittes kommt hinzu: Nicht nur der absolute Wohlstand einer Gesellschaft oder die wirtschaftliche Leistungsfähigkeit entscheiden über den Gesundheitszustand, ebenso wenig wie allein das für personenzentrierte Gesundheitsdienstleistungen ausgegebene bzw. investierte Geld. In hohem Maß scheint auch die relative Einkommens- und Vermögensverteilung Bedeutung zu haben. Die englischen Sozialforscher Richard Wilkinson und Kate Pickett legen diese Beobachtung eindrücklich in einem ihrer Bücher dar [5]. Länder mit einer geringeren Einkommensungleichheit wie Japan oder Norwegen weisen vergleichsweise geringe Gesundheits- und Sozialprobleme auf, Länder mit hoher Einkommensungleichheit wie die USA ungleich größere Probleme in diesen Bereichen.

Wie diese Effekte vermittelt werden, ist in weiten Bereichen noch offen und ist Gegenstand vielfältiger Forschungen, wie sie auch in dieser Ausgabe von „Das Gesundheitswesen“ wieder berichtet werden: Zur Kostenanalyse telemedizinsicher Maßnahmen, zu den Krankheitskosten bei Asthma und COPD, zur Fallzahlentwicklung der Brustkrebszentren in Nordrhein-Westfahlen, zur Langzeitverschreibung von Benzodiazepinen, zu subjektiver Gesundheit und sozialem Status, zum Wunsch- und Wahlrecht nach §9 SGB IX, zum Informationsbedarf niedergelassener Ärzte bei Ein- und Überweisungen und zur stationären Behandlung von Jugendlichen mit Alkoholintoxikation.

Zum Schluss sei noch die Frage gestellt, welche Alternativen als vielleicht wahrhaftigerer „Nerv der Dinge“ in Frage kommen. Hier kann nur eine unverbindliche Vorschlagsliste zur weiteren Prüfung durch die Leser vorgelegt werden. Vielleicht Ideen, die groß und schön genug sind, um das Innenleben zu prägen? Welches sich dann möglicherweise wieder nach außen wendet: in Form von Musik und anderen darstellenden oder bildenden Künsten? Lebenszeit in Gesundheit, die uns geschenkt wird, eine positive und hoffnungsvolle Sicht auf die Zukunft? Und vielleicht genau das Vertrauen zwischen Menschen und Gesellschaften, welches das Zwischentauschmittel Geld erst möglich gemacht hat und darüber hinaus auch selbst Grundlage ist für vieles von dem, für welches dieses Zwischentauschmittel sinnvoll eingesetzt werden kann - einschließlich der Gesundheit von Individuen und Bevölkerungen.

\section{Literatur}

1 Schenk B. 80000 Meilen und Kap Hoorn. Bielefeld: Delius Klasing Verlag; 1985

2 International Health Systems. Singapore: URL http://www.kaiseredu. org/Issue-Modules/International-Health-Systems/Singapore.aspx aufgerufen am 12.06.2013

3 Mackenroth G. Die Reform der Sozialpolitik durch einen deutschen Sozialplan. Berlin: Schriften des Vereins für Socialpolitik NF, Band 4. 1952

4 WHO. Macroeconomics and health: Investing in health for economic development. Report of the Commission for Macroeconomics and Health; Geneva: 2001

5 Wilkinson R, Pickett $K$. The Spirit Level. Why more equal societies almost always do better. London: Allen Lane; 2009 